\title{
Curcumol Induces Apoptosis in SPC-A-1 Human Lung Adenocarcinoma Cells and Displays Anti-neoplastic Effects in Tumor Bearing Mice
}

\author{
Qi-Ling Tang, 5\&, Ji-Quan Guo ${ }^{2 \&}$, Qi-You Wang ${ }^{3 \&}$, Hai-Shu Lin ${ }^{4}$, Zhou-Ping \\ Yang ${ }^{5}$, Tong Peng ${ }^{1}$, Xue-Diao Pan ${ }^{5}$, Bing Liu ${ }^{5}$, Su-Jun Wang ${ }^{5}$, Lin-Quan Zang ${ }^{5 *}$
}

\begin{abstract}
Curcumol is a sesquiterpene originally isolated from curcuma rhizomes, a component of herbal remedies commonly used in oriental medicine. Its beneficial pharmacological activities have attract significant interest recently. In this study, anti-cancer activity of curcumol was examined with both in vitro and in vivo models. It was found that curcumol exhibited time- and concentration-dependent anti-proliferative effects in SPC-A-1 human lung adenocarcinoma cells with cell cycle arrest in the G0/G1 phase while apoptosis-induction was also confirmed with flow cytometry and morphological analyses. Interestingly, curcumol did not display growth inhibition in MRC-5 human embryonic lung fibroblasts, suggesting the anti-proliferative effects of curcumol were specific to cancer cells. Anti-neoplastic effects of curcumol were also confirmed in tumor bearing mice. Curcumol (60 mg/ kg daily) significantly reduced tumor size without causing notable toxicity. In conclusion, curcumol appears a favorable anti-cancer candidate for further development.
\end{abstract}

Keywords: Curcumol - anti-proliferation - apoptosis - lung adenocarcinoma - tumor bearing mice

Asian Pac J Cancer Prev, 16 (6), 2307-2312

\section{Introduction}

Curcuma rhizomes are herbal remedies commonly used in oriental medicines such as Ayurveda and traditional Chinese medicine (Shishodia et al., 2005; Xia et al., 2005). During the past twenty years, curcumin, one of the key active substances of curcuma rhizomes attracted great interests in biomedical research (Shishodia et al., 2005; Anand et al., 2007). So far, thousands of research papers have been published with curcumin. The research interests have been extended to other active substances of curcuma rhizomes recently.

Curcumol, a sesquiterpene is another active substance of curcuma rhizomes (Carey et al., 2013; Chen et al., 2014). Similar to curcumin, curcumol also displayed a variety of beneficial pharmacological activities in preclinical studies, including anti-inflammation (Chen et al., 2014), anti-rheumatism (Wang et al., 2012; Wang et al., 2014; Yu et al., 2014) and anti-seizure (Ding et al., 2014) and hepato-protection (Chen et al., 2014). Moreover, the anti-neoplastic effects of curcumol have been well reported in various cell culture models (Wang et al., 2011; Zhang et al., 2011; Tian et al., 2012; Jing et al., 2013; Guo et al., 2014). However, the in vivo anti-cancer efficacy of curcumol remained unclear. To further examine the medicinal potential of curcumol, its anti-proliferative and apoptosis-inductive activities were assessed in SPC-A-1 human lung adenocarcinoma cells while the anti-cancer effects were examined in tumor bearing mice. To test whether the suppressive effects of curcumol are more specific to cancerous cells, the impact of curcumol on the growth of non-transformed cells was also attempted usingMRC-5 human embryonic lung fibroblasts as a model. To the authors' knowledge, this is the first report on the in vivo anti-neoplastic efficacy of curcumol in lung cancer model. Findings from this study will be useful to evaluate the application of curcumol as a chemotherapeutic agent.

\section{Materials and Methods}

\section{Chemicals}

Curcumol (racemate, purity $\geq 96.7 \%$ ) was obtained from the Haimen Desihang Co. Ltd. (Zhejiang, China), curcumol was dissolved in DMSO and dilute in PBS with $0.1 \%$ DMSO to incubate cells, dilute in normal saline with $1 \%$ DMSO to treat tumor bearing mice; RPMI and fetal bovine serum (FBS) were obtained from Gibco (New York, NY, USA). Methylthiazdyldiphenyltetrazolium bromide (MTT) was purchased from Genview

${ }^{1}$ College of Chinese Medicine, Guangdong Pharmaceutical University, ${ }^{2}$ Department of Respiration, Guangdong General Hospital, ${ }^{3}$ Department of Spine Surgery, The Third Affiliated Hospital of Sun Yat-Sen University, ${ }^{5}$ Novel Drug Screening Center, Department of Pharmacology, Guangdong Pharmaceutical University, Guangdong, China, ${ }^{4}$ Department of Pharmacy, Faculty of Science, National University of Singapore, Singapore \&Equal contributors*For correspondence: zanglq@163.com 
(California, USA). Hoechst 33258 kit, Cell cycle detection kit, Annexin V/PI kit was purchased from Kaiji Co. Ltd. (Jiangsu, China).

\section{Instruments}

Plate reader (BIO-RAD, California, USA) was used for the MTT assays. The apoptosis were quantified with a flow cytometer (Bechman-Coulter, California, USA), and the morphological analysis of cells was obtained with an inverted fluorescence microscope (Leica, Germany).

\section{Animals}

The in vivo study was carried out with compliance to the Guide for the Care and Use of Laboratory Animals (National Academy Press, 2011). The animal handling protocol was reviewed and approved by the Institutional Animal Care and Utilization Committee (IACUU) of the Experimental Animal Center of Guangdong Pharmaceutical University (approval no. SYXK (Yue) 2012-0125). Five-week-old BALB/c nude mice were obtained from Animal Experimental Center of Guangdong Province (Experimental Animals Certificate: SCXK (Yue) 2013-0002) (Guangdong, China). The animals were maintained in specific pathogen free animal facility with a $12 \mathrm{~h} / 12 \mathrm{~h}$ light-dark cycle at $22^{\circ} \mathrm{C}-24^{\circ} \mathrm{C}$ and $50 \%$ humidity. The animals had free access to food and water.

\section{Cells culture and MTT assay}

Human lung cancer cell line SPC-A-1 and human embryonic lung fibroblast cell line MRC-5 was obtained from Culture Biology of the Chinese Academy of Sciences (Shanghai, China). SPC-A-1 cells was cultured in RPMI1640 medium while MRC-5 cells in DMEM medium, both of them was supplemented with $10 \%$ fetal bovine serum. The cells were subcultured in a humidified atmosohere with $5 \% \mathrm{CO}_{2}$ at $37^{\circ} \mathrm{C}$ till reaching logarithmic growth phase.

Cells were seeded in a 96-well culture plate and treated with various concentrations of curcumol once for 24, 48 and $72 \mathrm{~h}$, respectively. After that, MTT assay was carried out using standard protocol and optical density (OD) was measured at $570 \mathrm{~nm}$ by using anenzyme-labeling instrument (BIO-RAD, USA). Cell proliferation was calculated, and the experiment was repeated 3 times.

\section{Apoptosis assay by hoechst 33258}

The SPC-A-1 cells and MRC-5 cells in logarithmic growth phase were seed in 24-well culture plate, and the cells were exposed to curcumol $(6.25,12.5,25,50$, $100 \mu \mathrm{M})$ for $48 \mathrm{~h}$. Then SPC-A-1 cells were stained by Hoechst 33258, and the changes in the nuclei of cells were examined and photographed by using a fluorescence microscope (Leica, Germany).

\section{Determination of cell apoptosis by annexin V/PI double staining}

Logarithmic growth phase of the SPCA-1 cells were seeded in 6-wells plate with density of $1 \times 10^{5}$ cells $/ \mathrm{ml}$. After cells were treated with various concentrations $(0$, $6.25,12.5,25,50,100 \mu \mathrm{M})$ of curcumol for $48 \mathrm{~h}$, the cells were doubled stained by Annexin V/PI apoptosis kit following to the instructions. Then the cell apoptosis rate was detected in a flow cytometer (Bechman-Coulter, USA).

\section{Determination of cell cycle by PI staining}

Logarithmic growth phase of the SPC-A-1 cells were seeded in 6-wells plate and treated with various concentrations $(0,6.25,12.5,25,50,100 \mu \mathrm{M})$ curcumol for $48 \mathrm{~h}$. Then the cells were exposed in RNase $(20 \mu \mathrm{g} / \mathrm{ml})$, and incubated with PI $(50 \mu \mathrm{g} / \mathrm{ml})$. Cell cycle distribution was analyzed using flow cytometry. The ModFit 3.1 program was used to determine the percentage of cells stalled at each phase of the cell cycle.

\section{Antitumor activity study of curcumol in vivo}

All the mice were injected in the right flank subcutaneously with SPC-A-1 cells $\left(2 \times 10^{6}\right.$ cells in 100 $\mu \mathrm{l}$ per site) at right forelimb armpit. When the tumors grew to approximate $4-5 \mathrm{~mm}$ in diameter, the tumor bearing mice were randomly allocated to 5 groups.

All the groups were observed for 13 days, and the tumor sizes were measured every two days. Tumor diameters were determined by using a Vernier caliper, Volume $=\left(\right.$ width $^{2} \times$ length $) / 2$. The mice were sacrificed on day 13 , the tumors were isolated and weighted. And the tumors tissues were collected for Hematoxylin-eosin staining.

Pathological observation of tumor tissue by hematoxylineosin staining

Each tumor of nude mice in different groups was fixed in $4 \%$ formaldehyde, dehydrated, paraffin-embedded, sectioned, and stained with Hematoxylin-eosin to observe changes in pathological cell morphology using a light microscope.

\section{Data analysis and statistics}

All experimental data are presented as Mean \pm Standard Error of the Mean (SEM). The value of 50\% inhibitory concentration $\left(\mathrm{IC}_{50}\right.$ ) was calculated with GraphPad Prism 6.05 (GraphPad Software, Inc., La Jolla, CA, USA). Two-tailed independent sample t-test was carried out with GraphPad InStat version 3.10 (GraphPad Software, Inc.). A $p$ value less than 0.05 was considered to be statistically significant.

\section{Results}

Anti-proliferative activities of curcumol

The growth-inhibitory effects of curcumol were examined in SPC-A-1human lung adenocarcinoma cells using cisplatin $(25 \mu \mathrm{M})$, a common chemotherapeutic agent for lung cancer as a positive control (Figure 1 (a), (b) \& (c)). Clearly, curcumol displayed time- and concentration-dependent anti-proliferative activities in SPC-A-1 cells. After $72 \mathrm{~h}$ incubation, the $50 \%$ inhibitory concentration $\left(\mathrm{IC}_{50}\right)$ on proliferation was found to be $32.7 \mu \mathrm{M}$. Cisplatin at $25 \mu \mathrm{M}$ possessed stronger growth inhibitory effect.

To find out whether the anti-proliferative activities of curcumol are more specific to cancerous cells, the 
impact of curcumol on the growth of MRC-5 human embryonic lung fibroblasts (non-transformed cells)was also attempted (Figure 1 (d), (e) \& (f)). Interestingly, curcumol did not have much growth inhibitory effect in MRC-5 and incubation with curcumol at $100 \mu \mathrm{M}$ for $72 \mathrm{~h}$ only led to less than $20 \%$ inhibition in comparison to the control. Clearly, the anti-proliferative activities of curcumol appeared to be more specific to cancerous cells.

\section{Morphological analysis of curcumol treated SPC-A-1} cells and MRC-5 cells

The morphology of curcumol treated SPC-A-1 cells and MRC-5 cells was observed after Hoechst 33258 staining. The nuclei of SPC-A-1 cells in control group (0 $\mu \mathrm{M}$ curcumol) emitted quite homogenous blue fluorescence (Figure 2 (a)), which could be explained by an even distribution of the chromatin in the nuclei. On the other hand, for some cells treated with curcumol, nuclear condensation, which emitting bright fluorescence was observed (Figure 2 (b), (c), (d), (e) \& (f)). Moreover, the cells treated with higher levels of curcumol, i.e. 25, 50 and $100 \mu \mathrm{M}$ had higher percentage of condensation. However, apoptotic bodies did not observed in MRC- 5 cells treated by curcumol (Figure 3 (a), (b), (c), (d), (e), (f)). Clearly, curcumol treatment could induce apoptosis in SPC-A-1 cells, while did not display apoptosis in MRC-5 cells.
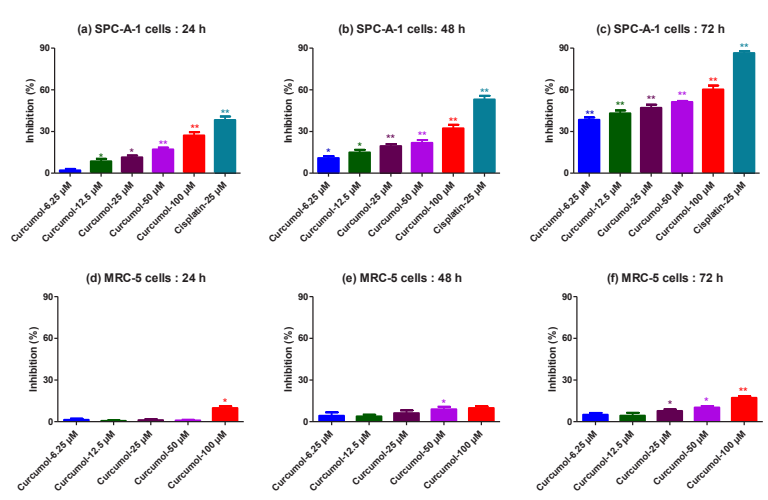

Figure 1. Anti-Proliferative Activities of Curcumol (a) in SPC-A-1 cells: $24 \mathrm{~h}$; (b) in SPC-A-1 cells: $48 \mathrm{~h}$; (c) in SPC-A-1 cells: $72 \mathrm{~h}$; (d) in MR-C-5 cells: $24 \mathrm{~h}$; (e) in MRC-5 cells: $48 \mathrm{~h}$; (f) in MRC-5 cells: $72 \mathrm{~h}$. Cell proliferation was assessed with MTT assay; columns represent mean values while error bars represent standard error of the mean (SEM) $(\mathrm{n}=3) ;{ }^{*} p<0.05$; $* * p<0.01$ vs Control group

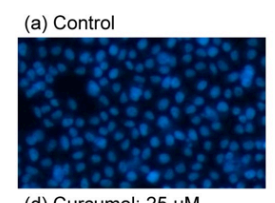

(d) Curcumol: $25 \mu \mathrm{M}$

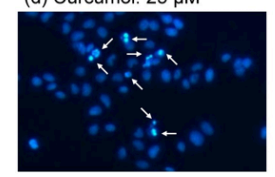

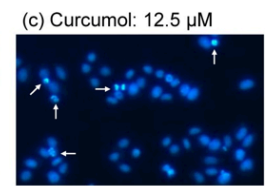
(f) Curcumol: $100 \mu \mathrm{M}$

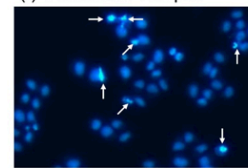

Figure 2. Morphology of SPC-A-1 Cells after $48 \mathrm{~h}$ Incubation of Curcumol at (a) $0 \mu \mathrm{M}$; (b) $6.25 \mu \mathrm{M}$; (c) $12.5 \mu \mathrm{M}$; (d) $25 \mu \mathrm{M}$; (e) $50 \mu \mathrm{M}$; (f) $100 \mu \mathrm{M}$. The cells were stained with Hoechst 33258 dye and observed with a fluorescence microscope (magnification: $\times 100$ ). Nuclear condensation is indicated with white arrows
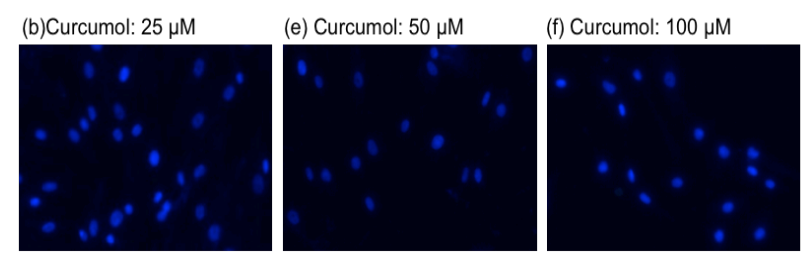

(a)Control (b) Curcumol: $6.25 \mu \mathrm{M}$
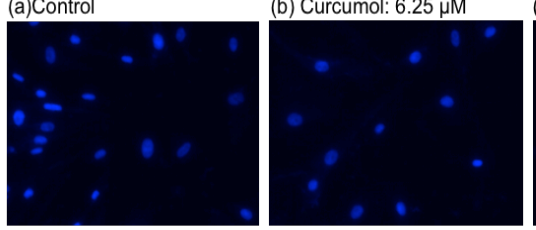

(c) Curcumol: $12.5 \mu \mathrm{M}$

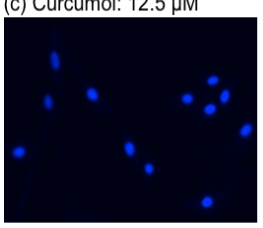

Figure 3. Morphology of MRC-5 Cells after 48h Incubation of Curcumol at (a) $0 \mu \mathrm{M}$; (b) $6.25 \mu \mathrm{M}$; (c) $12.5 \mu \mathrm{M}$; (d) $25 \mu \mathrm{M}$; (e) $50 \mu \mathrm{M}$; (f) $100 \mu \mathrm{M}$. The cells were stained with Hoechst 33258 dye and observed with a fluorescence microscope (magnification: $\times 100$ )
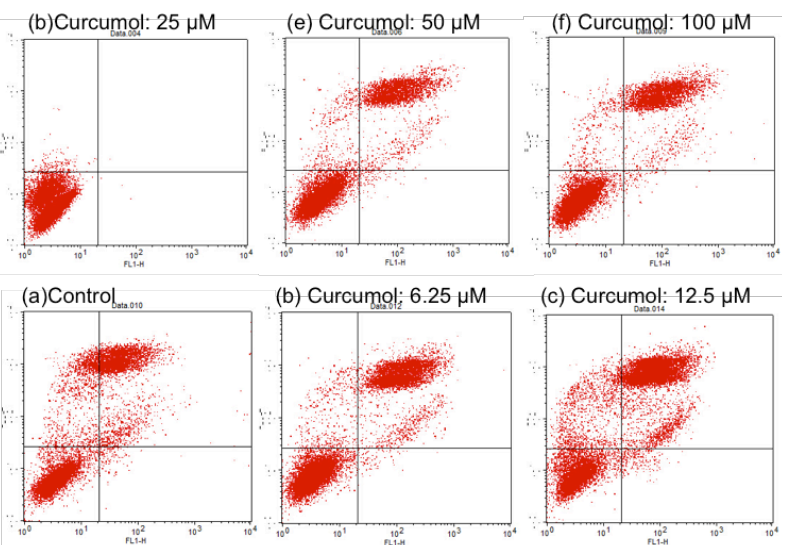

Figure 4. Apoptosis of SPC-A-1 Cells after 48h Incubation of Curcumol at (a) $0 \mu \mathrm{M}$; (b) $6.25 \mu \mathrm{M}$; (c) $12.5 \mu \mathrm{M}$; (d) $25 \mu \mathrm{M}$; (e) $50 \mu \mathrm{M}$; (f) $100 \mu \mathrm{M}$. The flow cytometry analysis using Annexin V/PI double staining to assess the apoptosis of SPC-A-1 cells treated with curcumol

Curcumol induced phase-arrest and apoptosis SPC-A-1 cells

The anti-neoplastic effects of curcumol were subsequently examined with flow cytometry. The cell cycle distribution was profiled after propidium iodide (PI) staining while the percentage of cells undergone apoptosis was quantified with Annexin V/PI double staining. The results are summarized in Table 1, the apoptosis of cells were observed in Figure 4.

Clearly, curcumol treatment led to cell cycle phase arrest at G0/G1 phase. Even at a concentration as low as $6.25 \mu \mathrm{M}$, curcumol increased the percentage of $\mathrm{G} 0$ / G1 phase SPC-A-1 cells. Interestingly, further increases in curcumol concentration up to $50 \mu \mathrm{M}$ did not lead to much change in the of phase distribution. In agree with the morphological observation, the flow cytometry analysis using Annexin V/PI double staining also confirmed that curcumol treatment induced apoptosis in SPC-A-1 cells. The apoptosis-inductive effect appeared to be concentration-dependent.

Curcumol displayed in vivo anti-cancer activities in tumor bearing mice

As curcumol displayed promising anti-neoplastic effects in in vitro studies, its in vivo anti-cancer efficacy 
Table 1. Curcumol Induced Cell Cycle Phase Arrest and Apoptosis in SPC-A-1 cells (a)

\begin{tabular}{llccc}
\hline Concentration $(\mu \mathrm{M})$ & \multicolumn{3}{c}{ Cell Cycle Distribution } & Apoptosis $(\%)$ \\
\cline { 2 - 5 } & $\mathrm{G} 0 / \mathrm{G} 1(\%)$ & $\mathrm{S}(\%)$ & $\mathrm{G} 2 / \mathrm{M}(\%)$ & $0.07 \pm 0.03$ \\
\hline Control & $54.93 \pm 0.53$ & $35.83 \pm 0.46$ & $9.24 \pm 0.18$ & $23.67 \pm 1.62 * *$ \\
6.25 & $59.75 \pm 1.10^{*}$ & $31.06 \pm 0.56^{* *}$ & $9.20 \pm 0.57$ & $25.72 \pm 0.97 * *$ \\
12.5 & $60.55 \pm 0.40^{* *}$ & $30.05 \pm 0.61^{* *}$ & $9.40 \pm 0.32$ & $28.81 \pm 0.85^{* *}$ \\
25 & $60.37 \pm 0.50^{* *}$ & $29.96 \pm 0.25^{* *}$ & $9.67 \pm 0.31$ & $35.43 \pm 0.85^{* *}$ \\
50 & $61.25 \pm 1.05^{*}$ & $29.85 \pm 0.93^{*}$ & $8.91 \pm 0.30$ & $52.18 \pm 4.59^{* *}$ \\
\hline 100 & $64.78 \pm 0.43^{* *}$ & $28.20 \pm 0.29 * *$ & $7.03 \pm 0.21^{* *}$ & \\
\hline
\end{tabular}

${ }^{\mathrm{a} D a t a}$ is presented as Mean $\pm \operatorname{SEM}(\mathrm{n}=3) ; * p<0.05 ; * *<<0.01 v s$ Control group.

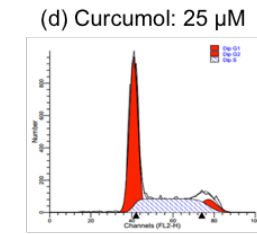

(a)Control

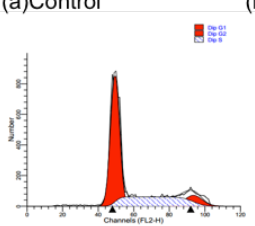
:

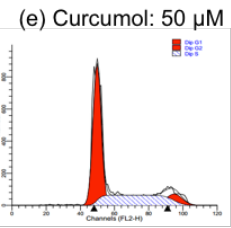

(b) Curcumol: $6.25 \mu \mathrm{M}$

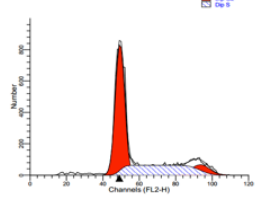

(c) Curcumol:12.5 $\mu \mathrm{M}$

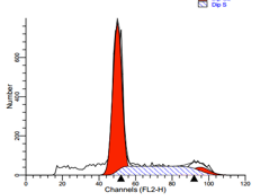

Figure 5. The Flow Cytometry Analysis Using Annexin Propidium Iodide (PI) Staining To Evaluate The Cell Cycle Distribution of SPC-A-1 Cells after $48 \mathrm{~h}$ Incubation of Curcumol at (a) $0 \mu \mathrm{M}$; (b) $6.25 \mu \mathrm{M}$; (c) $12.5 \mu \mathrm{M}$; (d) $25 \mu \mathrm{M}$; (e) $50 \mu \mathrm{M}$; (f) $100 \mu \mathrm{M}$

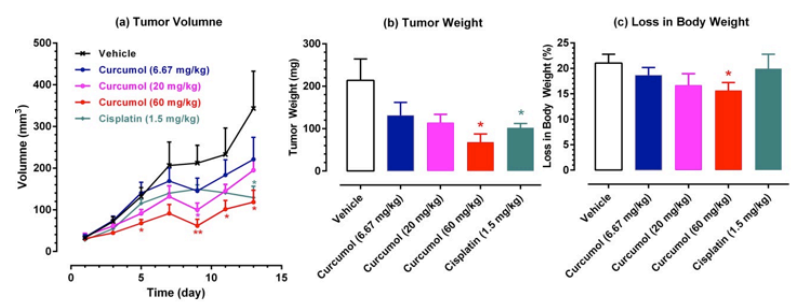

Figure 6. In Vivo Anti-cancer Efficacy of Curcumol. (a) tumor volume; (b) tumor weight; (c) loss in body weight. The anti-cancer efficacy of curcumol was examined tumor bearing mice. Tumor volume was measured every two days. On day 13, the mice were sacrificed; the tumors were isolated and weighted. Symbols or columns represent mean values while error bars represent SEM $(n=8$, except $n=7$ in the group of mice received curcumol at $60 \mathrm{mg} / \mathrm{kg}) ; * p<0.05 ; * * p<0.01$

was subsequently attempted in tumor bearing mice using cisplatin $(1.5 \mathrm{mg} / \mathrm{kg})$ as a positive control. Tumors were introduced through subcutaneous injection of SPC-A-1 cells $\left(2 \times 10^{6}\right.$ cells $)$ at right forelimb armpit. Drug treatment (curcumol or cisplatin) was started when the tumors diameter were about 4-5 $\mathrm{mm}$ and the drug treatment last for 12 days. At the end of the experiments, the mice were sacrificed and the tumors were isolated for further examination. The in vivo anti-cancer efficacy of curcumol is shown in Figure 6.

This mouse xenograft tumor model appeared to be fairly aggressive. For the mice in vehicle group, as they were not treated with anti-cancer agent (curcumol or cisplatin), the tumors progressed rapidly and the volumes increased by a few folds within 13 days (Figure 6 (a)).
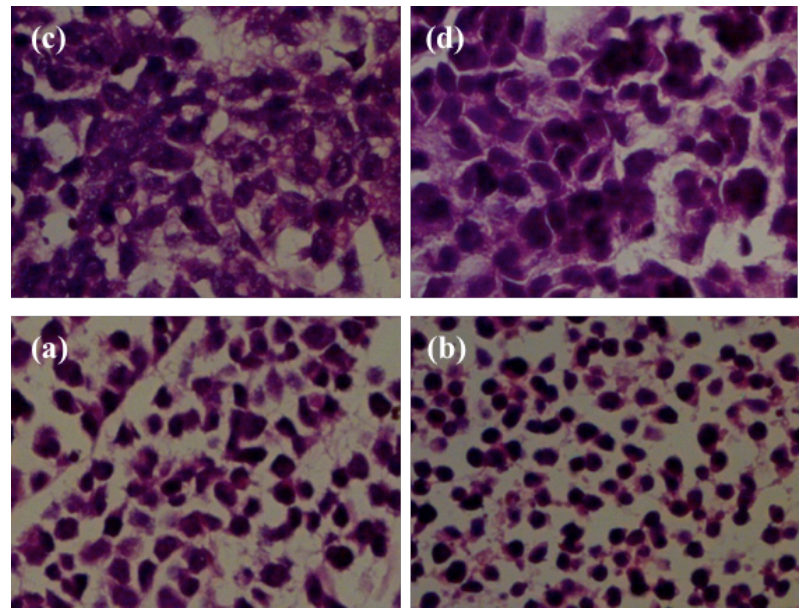

Figure 7. Histological Examination of the Tumors Treated with Curcumol. The malignant tissues were isolated from tumor-bearing mice, sectioned, stained with Hematoxylin-Eosindye and examined with a light microscope (magnification: $\times 400$ ). (a) Vehicle; (b) Curcumol: 6.67 $\mathrm{mg} / \mathrm{kg}$; (c) Curcumol: $20 \mathrm{mg} / \mathrm{kg}$; (d) Curcumol: $60 \mathrm{mg} / \mathrm{kg}$.

Moreover, all tumor bearing mice suffered from severe weight loss (Figure 6 (c)). As a positive control, cisplatin at the dose of $1.5 \mathrm{mg} / \mathrm{kg}$ displayed some anti-tumor effects. Although it decreased tumor volume and weight after 12 daily doses ( $p<0.05 v s$ vehicle group), it did not have protective effect in weight loss. As a chemotherapeutic agent, curcumol exhibited dose-dependent anti-cancer activities. Although the difference was statistically insignificant, the mice received low and intermediate doses of curcumol (6.67 and $20 \mathrm{mg} / \mathrm{kg}$ ) tended to have smaller tumors and suffered less from weight loss in comparison to the control group. When the dose of curcumol further increased to $60 \mathrm{mg} / \mathrm{kg}$, the in vivo anti-cancer cancer efficacy (using tumor volume, tumor weight and loss in body weight as endpoints) could be confirmed by statistical test ( $p<0.05 v s$ vehicle group). Interestingly, the anti-tumor activities of curcumol appeared to fast-acting. After 4 daily doses $(60 \mathrm{mg} / \mathrm{kg})$, curcumol was able to reduced tumor volume. The disease modifying effects of curcumol $(60 \mathrm{mg} / \mathrm{kg})$ was much faster than cisplatin $(1.5 \mathrm{mg} / \mathrm{kg})$.

The pathology of the isolated tumors was also examined. The histological observations are shown in Figure 7. The tumor cells isolated from mice received vehicle treatment are oval, large and polygonal; these cells also have hyperchromatic nuclei. On the other hand, the tumor cells isolated from mice treated with curcumol displayed nuclear condensation, chromatin gathered and 
cell shrinkage, suggesting they were undergoing apoptosis. Moreover, such signs appeared to be dose-dependent.

\section{Discussion}

According to World Health Organization, lung cancer is the leading cancer killer and it leads to 1.59 million deaths annually. As more than $80 \%$ of lung cancer cases are non-small cell lung cancer (NSCLC), we tested the anti-cancer activities of curcumol in SPC-1-A cell lines, a model for non-small cell lung cancer. Since cytotoxic combination chemotherapy, the first-line treatment for stage IV NSCLC includes platinum (cisplatin or carboplatin), we used cisplatin as the positive control.

For cytotoxic chemotherapy, the clinical toxicity and tolerability are one of the key issues. Therefore, it is of great interest to identify innovative chemotherapeutic agents that displayed selective cytotoxic effects to the malignant cells. Such selective chemotherapeutic agents may have better tolerability and safety profiles. The anti-proliferative effect of curcumol appeared to be more selective to cancer cells. For example, at 100 $\mu \mathrm{M}$, curcumol did not possess much growth suppression in MRC-5 human embryonic lung fibroblasts (nontransformed cells); however, it induced substantial apoptosis SPC-1-A cells. The selectivity of curcumol might lead to fewer side effects. Moreover, as curcuma rhizomes are commonly used as spices in cooking and curcumol is present in curcuma rhizomes at high level, the toxicity of curcumol would be limited. Such postulation was supported by pre-clinical observation in dogs (Zhang et al., 2007). Relatively low toxicity appeared to be a major advantage of curcumol and it may lead to better clinical tolerability/safety.

Pharmacokinetics plays an important role in drug development. The pre-clinical pharmacokinetics of curcumol has been attempted in dogs and rats after intravenous administration (Zhang et al., 2007; Zhao et al., 2010). Curcumol had moderate clearance in rats $(\sim 30 \mathrm{ml} / \mathrm{min} / \mathrm{kg})$ but relatively rapid clearance in dogs $(300 \sim 400 \mathrm{ml} / \mathrm{min} / \mathrm{kg})$. As a cancer chemotherapeutic agent, curcumol could be delivered through prolonged intravenous infusion. Therefore, a relatively rapid clearance is not a barrier to its clinical application. Moreover, its half-life was not too short (rats: 40 min, dogs: 2h). So, its pharmacokinetic s was unlikely a barrier to its clinical application.

The anti-tumor activities of curcumol were examined in tumor-bearing mice in this study. To our knowledge, this is the first report on its in vivo anti-cancer efficacy in NSCLC model. Curcumol displayed rapid onset diseasemodifying effects and such effects could be observed as early as after 4 daily treatments. The fast-acting antineoplastic effects appear to be another advantage of curcumol.

The remedies used in Traditional Chinese Medicine (TCM) have emerged as an important source for anticancer drug discovery. Various new anti-neoplastic drugs, e.g. Huachansu (water extract of Chinese toad skin) and Kanglaite (oily extract of coix seeds) have been developed and approved for the application in oncology. These TCM originated anti-cancer drugs sometimes potentiate the anti-neoplastic effects of some standard chemotherapeutic agents and therefore offer a superior clinical anti-cancer efficacy (Zhan et al., 2012; Xie et al., 2013). Currently, cytotoxic combination chemotherapy is used as the firstline treatment for stage IV NSCLC. As curcumol worked fast and might have good safety profile, it is of great interest to test curcumol in combination together with other standard chemotherapeutic agent (s) in NSCLC. Cisplatin / carboplatin plus curcumol could be the initial attempt in the future study.

In summary, curcumol exhibited time- and concentration-dependent anti-proliferative and apoptosis-inductive activities in SPC-A-1human lung adenocarcinoma cells and displayed anti-cancer effects in tumor-bearing mice. In conclusion, as its growthsuppressive effects were more specific to cancer cells and it might have good safety profile, curcumol appears as favorable anti-cancer candidate for further development.

\section{Acknowledgements}

This work was supported by The National High Technology Research and Development Program of China (863) (2012AA020304), and the Project of National Great New Drug Research and Development (2011zx09102001-31).

\section{References}

Anand P, Kunnumakkara AB, Newman RA, et al (2007). Bioavailability of curcumin: Problems and promises. Molec Pharmaceutics, 4, 807-18.

Carey AN, Fisher DR, Rimando AM, et al (2013). Stilbenes and anthocyanins reduce stress signaling in bv-2 mouse microglia. J Agr Food Chem, 61, 5979-86.

Chen G, Wang Y, Li M, et al (2014). Curcumol induces hsc-t6 cell death through suppression of bcl-2: Involvement of pi3k and nf-kappab pathways. European J Pharm Sci, 65, 21-8.

Chen X, Zong C, Gao Y, et al (2014). Curcumol exhibits antiinflammatory properties by interfering with the jnk-mediated ap-1 pathway in lipopolysaccharide-activated raw264.7 cells. Eur J Pharm, 723, 339-45.

Ding J, Wang JJ, Huang C, et al (2014). Curcumol from rhizoma curcumae suppresses epileptic seizure by facilitation of gaba (a) receptors. Neuropharmacol, 81, 244-55.

Guo P, Wang YW, Weng BX, et al (2014). Synthesis, anti-tumor activity, and structure-activity relationships of curcumol derivatives. J Asian Nat Prod Res, 16, 53-58.

Jing Z, Xie CY, Wu ZQ, et al (2013). Effects end mechanisms of curcumol beta-cyclodextrin compound on the proliferation and apoptosls of esophageal carcinoma cell line te-1. Zhongguo Zhong Xi Yi Jie He Za Zhi, 33, 85-89.

Shishodia S, Sethi G, Aggarwal BB (2005). Curcumin: getting back to the roots. Ann NY Acad Sci, 1056, 206-17.

Tian L, Dong J, Huang C (2012). Effect and mechanism of curcumol on angiogenesis activity of zebrafishes. Zhongguo Zhong Yao Za Zhi, 37, 1822-5.

Wang H, Fang Y, Wang Y, et al (2012). Inhibitory effect of curcumol on jak2-stat signal pathway molecules of fibroblast-like synoviocytes in patients with rheumatoid arthritis. Evid Based Complement Alternat Med, 2012, 746426.

Wang H, Wang Y, Jiang X, et al (2014). The molecular 


\section{Qi-Ling Tang et al}

mechanism of curcumol on inducing cell growth arrest and apoptosis in jurkat cells, a model of cd4 (+) t cells. Intern Immunopharmacology, 21, 375-382.

Wang J, Chen X, Zeng JH (2011). Effect of curcumol on proliferation and apoptosis of nasopharyngeal carcinoma cell line cne-2. Xi Bao Yu Fen Zi Mian Yi Xue Za Zhi, 27, 790-2.

Xia Q, Zhao KJ, Huang ZG, et al (2005). Molecular genetic and chemical assessment of rhizoma curcumae in china. $J \mathrm{Agr}$ Food Chem, 53, 6019-26.

Xie X, Huang X, Li J, et al (2013). Efficacy and safety of huachansu combined with chemotherapy in advanced gastric cancer: A meta-analysis. Med Hypotheses, 81, 243-50.

Yu M, Chen X, Lv C, et al (2014). Curcumol suppresses ranklinduced osteoclast formation by attenuating the jnk signaling pathway. Biochemical Bioph Res Co, 447, 364-370.

Zhan YP, Huang XE, Cao J, et al (2012). Clinical safety and efficacy of kanglaite (r) (coix seed oil) injection combined with chemotherapy in treating patients with gastric cancer. Asian Pac J Cancer Prev, 13, 5319-21.

Zhang R, Wang BJ, Zhao HL, et al (2007). Determination of curcumol in plasma by hplc-ms/ms method and its pharmacokinetics in beagle dogs. Үaо Хие Хие Ваo, 42, 973-7.

Zhang W, Wang Z, Chen T (2011). Curcumol induces apoptosis via caspases-independent mitochondrial pathway in human lung adenocarcinoma astc-a-1 cells. Med Oncol, 28, 307-14.

Zhao X, Liu YL (2010). Determination of curcumol in rat plasma by capillary gas chromatography with a hydrogen flame ionization detector. Biomed Chromatography, 24, 347-50. 